\title{
Diagnóstico de las universidades privadas y su contribución al desarrollo de El Salvador
}

\author{
Blanca Ruth Orantes
}

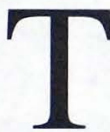

odos los fenómenos que surgen en la sociedad, son el efecto de la dinámica socioeconómica y política. El surgimiento de universidades privadas obedeció a la necesidad de brindar más opciones a los aspirantes a la educación superior. Lo anterior constituyó la premisa para iniciar un diagnóstico general de las universidades privadas, que va desde su génesis hasta su crecimiento y su situación actual. En este contexto se inicia este proyecto de investigación como producto del quehacer investigativo de la Universidad Tecnológica de El Salvador.

Si bien es cierto las universidades privadas son entes educativos, en algún momento las ven como empresas privada, no obstante estas instituciones, independiente de cómo las observen, tienen el compromiso que va desde formar a las nuevas generaciones hasta dar aportes a la sociedad en inves- tigación y proyección social. Lo anterior las hace vulnerables a la crítica que, siendo constructiva, puede ser un aporte para el comportamiento y desarrollo de estas universidades. Buscando respuestas se realizó este estudio con una visión académica constructiva. Para ello se accedió a las fuentes de información primarias y secundarias. Las primarias permitieron acceder a los sujetos de estudio, denominando algunos de ellos informantes clave, buscando por medio de las entrevistas obtener la percepción y visión de personal ejecutivo de las universidades privadas. Estos sujetos se seleccionaron dentro de las universidades con cargos directivos o ejecutivos, así como personajes de la palestra política, educativa y económica de otros sectores. Los resultados de las entrevistas se vaciaron en una ma- 
triz que se puede apreciar cuando se consulte el informe final, pero a la vez sirvieron de base para análisis en el marco de referencia y la formulación de algunas de las recomendaciones. En la búsqueda de respuestas siempre como fuentes primarias, se entrevistaron a graduados de las universidades privadas para obtener su percepción y perfil de graduado, como producto de estas instituciones. Las fuentes secundarias constituyen el soporte teórico que permiten describir los hechos, buscando un orden lógico y coherente al contexto en que se desarrolla el presente estudio.

\section{Contexto delasuniversidades privadas}

En el contexto sociocultural y político que surgieron las universidades privadas, se dieron movimientos sociales y políticos que desencadenaron en nuestro país una polarización que llevó a una guerra civil que confrontó a varios sectores de la sociedad salvadoreña. La conflagración sin duda afectó a la única universidad nacional, creando temor en estudiantes y padres de familia. Lo anterior permitió que muchas universidades privadas surgieran $\mathrm{y}$, ante este fenómeno, se observó la necesidad de regularlas.

Esta regulación llevó a cambios organizacionales positivos para la educación superior, lo cual ha beneficiado al sistema y a muchas universidades, que han tenido que adecuar sus condiciones, su sistema interno, a las exigencias de la ley, pero que a la vez les ha permitido mejorar y prepararse para competir. Algunas de estas universi- dades, que lo han logrado competir en mejores condiciones están enfatizando en la calidad académica y en mejorar los sistemas de investigación y proyección social.

Las universidades privadas que existen en la actualidad, sin duda han tenido que enfrentar múltiples problemas, superado algunos de ellos y en proceso de superar otros. De ahí la importancia de una investigación que identifique los aportes que han dado al desarrollo de El Salvador, y señalar los aspectos positivos de estas y la contribución a los diferentes sectores.

El estudio pretende, por una parte, mostrar los esfuerzos de las universidades privadas en materia educativa; y por otra, en capital humano. Se visualizan algunas necesidades en las que puedan ser apoyadas por los diferentes sectores que incluyen al Estado y al sector productivo. En cuanto al capital humano que se forman en las universidades privadas, que no es más que el producto, se pretende exponer las circunstancias en que se formaron, tratando de explorar fortalezas y debilidades, que tomado positivamente, pueden constituir un baremo para medir algunos puntos sensibles en la formación de los universitarios.

Algunas universidades privadas en El Salvador buscan mantener su autorización, otras buscan acreditarse, con el objeto de lograr las competencias en el nuevo contexto y los nuevos escenarios. Lo anterior les ha permitido mejorar sus condiciones, atender eficazmente a los estudiantes, mejorar el currículo, los servicios administrati- vos, entre otros. Pero, sobre todo, ampliar su campo de acción, adaptándose a las nuevas modalidades. En este mismo orden la educación a distancia, que ya es uno de los instrumentos principales en la educación superior, es una nueva carta de competencia para las universidades, pero a la vez puede ser también un reto. Tal como lo manifestó Larry Kenem ${ }^{1}$ en 1999 , la educación a distancia en aquella época se visualizaba como uno de los principales instrumentos para popularizar aún más la educación. El problema que se observa en la actualidad es que, si bien es cierto resulta una ventaja, se ha transnacionalizado, ya que los cursos son desarrollados para un mercado y los costos comienzan a ser recuperados en el mercado transnacionalizado a partir de muy pequeñas inversiones, y cada vez más con una pequeña inversión adicional estos cursos pueden ser ofrecidos en otros países a muy bajos costos. Cuando se comparan precios, dijo Kenem, con los servidores locales de educación, no es posible competir en el costo. Esto es más evidente en los cursos transnacionalizados de uso de software propiedad de los grandes productores de informática de Estados Unidos. En el 2007 este país fue el más grande exportador de educación a distancia.

Sin embargo, aún existen dos aspectos que expertos ${ }^{2}$ señalan hay que apuntarles fuerte: actualización de los contenidos y la formación de los docentes. Todo con el objeto de alcanzar y mantener la calidad académica en la educación superior. Ambos aspectos están directamente vinculados a potenciar la formación de los educandos

\footnotetext{
KENEM, Larry. La globalización de la educación en las Américas. Conferencia IDEA. Quito. Septiembre-octubre 1999. Citado por Marco Raúl Mejía J. "Leyendo las políticas educativas de la globalización", presentación en el panel sobre reformas educativas en América Latina en el XX Congreso de la CIEC, Santiago de Chile, Enero 8-14 de 2004.
}

2 Visión para el mañana. Imprescindible elevar la calidad académica. Guatemala, 26 de octubre 2007. 
y futuros profesionales. "Los profesores son la pieza clave"; en palabras de un funcionario coreano citadas por The Economist: la calidad de un sistema educativo no puede ser mejor que la de sus profesores. "Según estudios hechos en Estados Unidos añade la revista si se toman alumnos de capacidad media y se les encomienda a profesores del $20 \%$ mejor valorado del cuerpo docente, acaban dentro del $10 \%$ con mejores notas; si se les pone con profesores del 20\% más bajo, acaban entre los de peores notas"3. Este punto es un aspecto medular, porque tanto la educación a distancia como la internacionalización de las universidades, exigen estándares de calidad a escala nacional, centroamericana e internacional. Las universidades tendrán que renovar su planta docente e intensificar sus programas de capacitación con el objetivo de actualizar y modernizar al docente en sistemas de enseñanzaaprendizaje apegados a la realidad. Se tiene que abandonar esos métodos de enseñanza de simples transmisores del conocimiento y pasar a la creación e innovación de los mismo.

Otro fenómeno que se encuentra en el entorno de las universidades es la internacionalización universitaria. Si se observan en el pasado las relaciones internacionales eran un elemento de cierta importancia, pero casi optativo, que permitía intercambio académico y colaboración en forma más o menos esporádica, y dependiendo crucialmente de difíciles y costosas comunicaciones. Todo ha cambiado: las universidades precisan de activas y permanentes relaciones internacionales porque el mundo se ha globalizado. Los estándares de calidad deben obedecer a patrones internacionales, y son muchos los estudiantes en todo el mundo que esperan continuar sus estudios en otro país y con un distinto idioma, ya sea que provengan de países desarrollados o en vías de desarrollo. Al respecto se observa muy bajo intercambio de estudiantes salvadoreños en el exterior. En muchas ocasiones el idioma inglés ha sido el mayor obstáculo, por lo que se requiere preparar estudiantes competitivos para gozar de los beneficios del intercambio. Lo anterior no solo significa el dominio de un segundo idioma, sino también elevar la calidad académica a su máxima expresión que, además de reflejar un CUM, ${ }^{4}$ muestre otras competencias en su desempeño por medio de las habilidades y destrezas técnicas y académicas.

Por otra parte, las necesidades del mundo del trabajo han incentivado el desarrollo de prácticas profesionales y postgrados en otros países, mientras que muchos académicos que aprecien su formación deben preocuparse de obtener un grado de alguna universidad integrante de algún ranking respetable y reconocido. No obstante, no todos pueden trasladarse a las sedes de estas universidades. Por ello en la actualidad se observa como surgen convenios para ofrecer los programas de maestrías y doctorado en las universidades de El Salvador que, dicho sea de paso, deben ser convenios bajo los principios de reciprocidad, igualdad y equidad, en el sentido que el beneficio tenga carácter tripartido:

- Universidad extranjera

- Estudiantes

- Universidad privada nacional.
Los programas de estudios deben contener un importante componente internacional, y los criterios de evaluación de la actividad deben responder a patrones nacionales e internacionales, como asimismo lo deben ser la estructura de los programas que deben garantizar efectivas posibilidades de intercambio, un punto que las universidades deben revisar. Este último aspecto no se observa en la actualidad, por lo que es urgente se revisen los programas bajo los criterios de estándares de calidad internacionales.

En la realidad universitaria actual existen convenios con otras universidades, pero no se observan beneficios significativos para las universidades locales y los estudiantes nacionales. Como ejemplo se pueden citar los altos costos en los postgrados, que asumen las universidades privadas nacionales. $\mathrm{Y}$ como efecto de cascada a veces no se llenan las plazas, por lo que dejan pérdidas para las universidades locales, además de no permitir acceder a los programas a muchos salvadoreños interesados en superarse.

\section{Resultados de instrumentos aplicados a graduados}

$\mathrm{Al}$ preguntarles por qué seleccionaron esa universidad, las tres opciones preferentes fueron: $28 \%$ respondió que por prestigio; $25 \%$ por un abanico de características, tales como prestigio, bajo costo de estudios, ubicación geográfica y horarios accesibles; $20 \%$ por la ubicación geográfica. Lo que muestra algunos de los aspectos del porqué los estudiantes prefieren las universidades privadas independiente de la institución que provengan, sea pública

Los tres secretos de la calidad de enseñanza. The Economist. Aceprensa.com, 25 octubre 2007.

4 Coeficiente de Unidades de Mérito (CUM) Art. 7. Ley de Educación Superior. República de El Salvador. 
o privada. El 53\% proviene del sector privado y $47 \%$ del sector público, demostrando con ello que la preferencia por las privadas no solo es de las personas adineradas. Dicho sea de paso, que también dentro de las privadas hay instituciones con diversa oferta financiera, acorde a la capacidad económica de cada estudiante. Se puede corroborar con las respuestas al sondeo que se hizo en relación a la institución de procedencia, en donde $53 \%$ de graduados por las universidades privadas estudiaron su bachillerato en instituciones privadas, y $47 \%$ en públicas.

Se les preguntó si tenían trabajo antes de graduarse, con el objeto de observar su situación. Así, 39\% manifestaron que tenían trabajo a tiempo parcial; $33 \%$ a tiempo completo y $26 \%$ no trabajaba. Lo anterior sustenta la aseveración que muchos estudiantes con responsabilidades laborales prefieren las universidades privadas por los horarios convenientes y su ubicación accesible.

Indagando si se cambió de carrera, $36 \%$ manifestaron que nunca; $34 \%$ dijeron que una vez; $19 \%$ dos veces y $11 \%$ tres veces. Es notable que en su mayoría se mantuvieron en la carrera $\mathrm{o}$, al menos, solo se cambiaron una vez. Y como sucede hasta en la UES, hay una minoría que se cambia hasta tres veces. Pero en todos los casos ya hay reglas internas y externas establecidas.

En nuestro país un indicador de éxito profesional puede medirse por el nivel de ingresos que se recibe, $y$ que permite llevar una vida digna. En este caso los porcentajes más representati- vos que arrojó la situación actual de ingresos de los profesionales graduados en universidades privadas, fueron: $24 \%$ de profesionales tienen un salario de $\$ 701$ a $\$ 1.000$; el $22 \%$ de $\$ 501$ a $\$ 700$; el $21 \%$ de $\$ 300$ a $\$ 500$. Los demás porcentajes son minoritarios. Sí llamó la atención que $13 \%$ gana menos de $\$ 300$. Puede observarse que la mayoría no percibe salarios mayores de $\$ 1.000$. Algunos de ellos aducen que no es necesariamente por incapacidad, sino más bien a los "bajos salarios característica de nuestro país. Éstos, como bien se mencionó antes, no aseguran una vida digna a las familias"5. Sin embargo, el buen salario es una señal de éxito en los profesionales, podría decirse que hay más de la mitad que no ha conseguido ese éxito. Aunque las causas sean diversas, que van desde los bajos salarios, baja competencia del perfil profesional y desempleo, hacen que muchos profesionales opten por trabajar en "lo que sea".

Respecto a las capacidades adquiridas durante la formación universitaria, se pudo determinar las principales que, según ellos adquirieron en la "U": $16 \%$ manifestaron que el trabajo en equipo; $14 \%$ la responsabilidad, y $9 \%$ la capacidad de comunicarse, así $8 \%$ la resolución de problemas. Mientras $11 \%$ que ninguna. El restante porcentaje no es representativo en los indicadores sondeados. Por lo que se puede observar, muchos de los estudiantes salen con baja capacidad en su perfil académico, pues el profesional puede aumentarlo ya en su vida laboral. Resulta importante diferenciar el perfil académico del profesional. Pues cuando los estudiantes se gradúan salen con un CUM académico que no nece- sariamente refleja su perfil profesional, pues este último se fortalece con la práctica.

Con el objeto de sondear también los aspectos cognoscitivos, se les preguntó acerca de la significancia de lo aprendido, y respondieron de la siguiente forma: $39 \%$ manifestaron que en mucho les ha servido los conocimientos, habilidades y destrezas adquiridas en la carrera para aplicarlas al trabajo; $29 \%$ dijeron que algo, $22 \%$ moderadamente y $10 \%$ respondieron que no, en absoluto. Por ello la valoración que dan a la utilidad de lo aprendido lo califican de regularmente $32 \%$ y $30 \%$ bastante, y solo $24 \%$ los utilizan completamente. Nos surge el interrogante: ¿A qué aspectos le dan énfasis los maestros que no se ha logrado enseñar $100 \%$ lo que se utilizará en el ambiente laboral? Lo que respondieron los profesionales graduados: $64 \%$ dijeron que enfatizaban en lo teórico sin aplicación práctica; $26.8 \%$ enfatizaban más en lo práctico, estos fueron los porcentajes representativos. Hay que tomar en cuenta que se encuestó a graduados de hace entre 1 a 10 años, por lo que se puede decir que poco se ha evolucionado en el proceso enseñanza-aprendizaje. En este sentido, se observa la necesidad urgente de equilibrar la teoría con la práctica en el aula. No significa que lo teórico no tenga su valor, pero cada vez más se hace necesaria su aplicabilidad en escenarios posibles, y mejor aún sería aumentar los programas de pasantías con las empresas e instituciones de gobierno. Así también, aumentar la planta docente que se desarrolla en la práctica, pues son muchos los docentes que se dedican a esta actividad por no tener

5 LARA LÓPEZ, Edgar y Rubio Jovel, Silvia. Cuaderno de análisis y propuestas “¿Tenemos buenos empleos? Impacto de las políticas económicas en los buenos empleos de El Salvador." Fundación Nacional para el Desarrollo -FUNDE. Reporte de País para el proyecto WFD-2, preparado para Global Policy Network. San Salvador, El Salvador, Centro América. P-11. 
otra oportunidad laboral, pero que en su vida han ejercido su profesión.

En cuanto a los valores transmitidos en las universidades: $40 \%$ dijeron que casi nunca se los transmitían; $24 \%$ dijeron que casi siempre y $25 \%$ algunas veces. Esto muestra debilidad en cuanto a la transmisión de valores, al menos en las generaciones de ex estudiantes que opinaron. Aunque los que opinaron que sí se les transmitieron valores, mencionaron tres relevantes: cooperación, integridad y rectitud, y además creatividad. Se menciona lo anterior porque desde los últimos dos años existen instituciones que ya involucran, dentro de las competencias, los valores más importantes que conduzcan a un cambio de actitud en sus estudiantes, considerando a estos futuros profesionales y agentes de cambio.

A propósito, cuando se les preguntó si se consideraban un agente de cambio a partir de su inserción al ámbito profesional, manifestaron lo siguiente: solamente algunas veces se consideran agente de cambio dijeron $43 \%$ de profesionales; $24 \%$ que casi siempre; $21 \%$ a menudo, y $12 \%$ casi nunca. Por lo que se considera que un alto porcentaje no se prepararon como agentes de cambio.

En cuanto a la sensibilidad y solidaridad social, se les preguntó si participan en proyectos de su comunidad o sociales. Las respuestas fueron: $46 \%$ casi nunca; $19 \%$ a menudo; $19 \%$ casi siempre, y $16 \%$ algunas veces. Aquí es evidente una baja sensibilidad y solidaridad en los profesionales encuestados.

No obstante, los sujetos de estudio consideran que las cualidades que les distinguen en el mercado laboral dentro de las principales son: cooperación con $26 \%$; la integridad y rectitud con $22 \%$, la creatividad con $20 \%$, y el servicio: $19 \%$. Se observaron debilidades en muchos por el bajo porcentaje en cuanto a lo proactivo, que se considera una cualidad importante en el ámbito laboral, puesto que puede convertirlos en motores de cambio en los sectores productivos.

Con el objeto de sondear la opinión de los encuestados de cómo se definirían como producto de las universidades privadas, se calificaron así: $48 \%$ dijeron que bueno; $32 \%$ regular; $12 \%$ deficiente y solo $8 \%$ excelente. Lo que puede tomarse como un indicador contundente de que se necesita mejorar el proceso; y para un producto mejor, pues si salen con baja autoestima o conscientes de su baja competencia, sin duda su inserción al ámbito laboral les traerá muchas dificultades.

A partir de su experiencia, los profesionales entrevistados consideran que las universidades deben reforzar las siguientes áreas: $27 \%$ dijeron que el inglés es una carta de competencia prioritaria; $27 \%$ opinaron que la administración y gerencia es de mucha utilidad en cualquier carrera, $23 \%$ el conocimiento y habilidad en nuevas tecnologías, y $12 \%$ creación de fuentes propias de trabajo; le sigue diseño y ejecución de proyectos con $11 \%$. Estos datos urgen necesidades detectadas ya en el ámbito laboral y que podrían hacer más competente al recurso humano que se forma en las universidades privadas.

\section{Algunas conclusiones}

Las universidades privadas nacen en un contexto histórico para suplir una necesidad educativa urgente. El crecimiento implica aprender, equivocarse, corregir, entre otros aspectos. No obs- tante, en sus vivencias las universidades privadas se han posicionado en el mercado educativo, un mercado que no solo trae beneficios, sino también aportes que han permitido a la sociedad salvadoreña mejorar ciertas condiciones y desarrollarse.

El Estado es un garante de los derechos de la población, por tanto debe garantizar la educación. Si se cuenta con un Estado descentralizado y moderno, producto de la misma dinámica social, es indudable que el sistema como tal permite que los sectores privados suplan algunas de las necesidades de la sociedad, tal es el caso de la educación superior. Hasta este momento se realiza una reflexión apolítica, centrada en una realidad en donde el Estado, por medio del MINED, es el garante; $y$ las universidades privadas deben obedecer la ley, y así lo hacen, caso contrario desaparecen. Por ello la evolución de las universidades ha respondido a ponerse a tono con la ley; aunando esfuerzos tratan de mejorar la calidad académica y no solo lo que dice el MINED sobre la base de criterios dados, sino también bajo un concepto más amplio, y así competir y hacerse notar como agentes de desarrollo. Ese es el reto, pues aún se está en el camino, ya lo han señalado los entrevistados, pero más enfáticamente los profesionales graduados de las universidades privadas que ya han enfrentado el mercado laboral.

Se encuentran muchas debilidades, pero también oportunidades de superarlas. Sin duda hay que apostarle decididamente a la mejora de la planta docente, lo que significa especialización, pedagogía y sensibilidad. El docente universitario tiene también el compromiso de formar, al igual que el padre. En la universidad es un deber formar profesionales competitivos, 
pero que no se vuelvan miopes ni apáticos ante los problemas que enfrenta nuestra sociedad; y para satisfacer las necesidades de recurso humano del sector productivo para el que se está formando al estudiante. La mejora en la planta docente significa actualización tecnológica, manejo de un segundo idioma, por lo menos, pero sobre todo vocación por formar un recurso humano de calidad y agente de cambio. La premisa es que se debe formar para la vida, no para un examen. El producto de las universidades privadas (los graduados) debe mejorarse de una forma integral, pues el sondeo de opinión refleja un producto no acabado y esto implica un gran compromiso.

En cuanto a los graduados, los bajos salarios que algunos manifiestan pueden tener varias causas, entre ellas, la baja competitividad; otra, la saturación de carreras, como es el caso de Derecho y Administración de Empresas, que fueron algunos de cuyos graduados presentaron bajos ingresos. Por supuesto, la crisis económica que tiene sus causas también en la estructura, que nos traslada a una situación de fondo y que no es objeto de este estudio.

Se ha enfatizado que las universidades privadas requieren de apoyo, principalmente en los proyectos orientados a la sociedad. La investigación tecnológica y la investigación acción, son rubros que requieren recursos financieros altos, pero urgentes de implantar en aquellas que aún no lo hacen.

Por ello las universidades deben invertir, en primera instancia, y luego gestionar alianzas con las empresas, organismos internacionales y universidades o instituciones extranjeras, en condiciones de igualdad, para que en realidad se vislumbren beneficios recíprocos.
Las universidades privadas han aportado al desarrollo de El Salvador, en un primer orden atendiendo a los clientes del sector educativo que, según lo manifiesta la Constitución, no es exclusivo del Estado. Los clientes que son estudiantes de diversos estratos sociales, han tenido la oportunidad de invertir en su educación, logrando con ello alcanzar una parte fundamental de su proyecto de vida, cual es una profesión; esto sería en segundo orden. En tercero, la investigación en las universidades privadas, si bien ha tenido divulgación débil, ha contribuido en alguna medida a crear debate nacional y a llamar la atención de algunos sectores de la sociedad y, en algún momento, a despertar interés en algunos expertos. Aunque algunas opiniones sean críticas, se deben tomar en consideración para la mejora continua.

La proyección social ha tenido presencia, y muchas son las universidades privadas que se han hecho sentir y han logrado consolidar alianzas con diversos sectores, sean públicos o privados A manera de ejemplo, el caso de los convenios celebrados por diversas universidades con la Corte Suprema de Justicia, que permite, por medio de los socorros jurídicos, atender a la población de escasos recursos, brindándoles asesoría jurídica. Son estudiantes los que participan activamente, realizando sus prácticas. Se ve claro cómo la sociedad se beneficia con este servicio: los estudiantes con sus prácticas profesionales; el Estado y la sociedad recibe contribución en materia de atención jurídica y disminución de la mora judicial.

Lo anterior constituye a grandes rasgos algunas de las contribuciones que las universidades dan a la sociedad salvadoreña. El camino al desarrollo por supuesto es difícil; pero se está buscando, y las universidades privadas no son ajenas a ello.

\section{Algunas recomendaciones}

En los proyectos educativos deben incluirse valores de la calidad tales como el respeto, la equidad, la igualdad, el sentido de pertenencia, la solidaridad y la honestidad. Y debe ser el docente el principal portador, enseñando con el ejemplo.

Se deben revisar los contenidos curriculares, pero en forma consultada con los sectores afines (en que se desarrollará el futuro graduado), con el objeto de incluir las competencias sobre la base de escenarios inmediatos, mediatos y a largo plazo, Se estaría con ello más cerca de enviar el "producto humano" que las empresas e instituciones del sector productivo requieren.

Se debe buscar la excelencia de los planes académicos y en los proyectos investigativos de la calificación postgradual, y en las competencias pedagógicas de los docentes y de la formación de profesionales aptos para la resolución de problemas propios del contexto socio-económico y político del país, en correspondencia con las exigencias de la internacionalización del conocimiento. Todo lo anterior implica revisar los contenidos curriculares, metodologías y prácticas de enseñanza-aprendizaje en su totalidad y como etapas naturales del proceso educativo.

Los valores deben fomentarse también en los docentes, generar las aptitudes

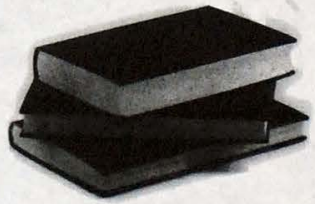

Junio $2008, \mathrm{~N}^{\circ} .40$ 
positivas y una tendencia a la indagación permanente; todo como un conjunto integral de un perfil renovado del docente. Hay que prodigar con el ejemplo.

Deben actualizarse los perfiles de graduados, preparar agentes de cambio. La principal misión de la "U" como la llaman muchos estudiantes y graduados es formar profesionales que por medio del conocimiento generen cambios cualitativos en su entorno, que permitan su desarrollo personal, institucional y a escala de país. Ya que las universidades, aunque privadas, el Estado les confiere responsabilidad social al permitirles su existencia, por ello son extensión del quehacer social, económico y político de El Salvador en la búsqueda de la paz y el bienestar social.

Las universidades mejor posicionadas deben trabajar en un examen de admisión no discriminativo, porque se les quitaría la práctica democrática a las universidades privadas. No obstante, este examen puede permitir identificar fortalezas y debilidades en los estudiantes que ingresan, así como orientarles en la carrera para la que tienen aptitud y evitar con ello fracasos. Estas universidades, que son las llamadas a prevalecer en un escenario de país "en proceso de desarrollo", estarán obligadas a generar un mayor valor agregado profesional, independientemente de "la calidad de insumo" que reciban de la educación media.

Los interciclos deben aprovecharse, más que para adelantar materias, para fortalecer algunas debilidades y darles cursos de nivelación en algunas competencias generales (expresión oral y escrita, matemática, investigación, refuerzos de inglés y lógica, entre otros) de carácter obligatorio a los estudiantes que tienen bajo CUM. Asimismo, el MINED debe exigir más calidad desde la primaria, pues muchos de los problemas de los estudiantes universitarios vienen desde los niveles más básicos.

Si tenemos una sociedad consumista, se debe trabajar por una campaña intensa y extensa que potencie el consumo del servicio educativo en la población, para que invierta en un proyecto de vida en vez de en otros productos suntuarios, ya que el nuestro es un país de bajo nivel de ingreso por habitante.

La acreditación para las universidades debe ser obligatoria, pues si esta refleja un nivel de calidad, sin duda debe ser el instrumento de medición único para alcanzar la calidad académica.

En materia investigativa, las universidades privadas necesitan dar también un salto de calidad, en cuanto a inversión en recursos e iniciación de la investigación aplicada. Los resultados de los proyectos de investigación deben volcarse hacia la sociedad. Existen tantos problemas en la sociedad salvadoreña, que van desde lo social hasta la bien marcada dependencia tecnológica, que nos ubica como un país subdesarrollado. La proyección social debe estar, sin duda, vinculada a las otras dos funciones: la docencia y la investigación; pero se requieren también muchos recursos, que las universidades deben considerar buscar en organismos internacionales, empresa privada y el Estado.
De hecho, si se cumple con los niveles de calidad en las tres funciones principales de toda universidad, se estaría preparado para competir a escala nacional e internacional. En este último escenario, se requiere la alianza con otras universidades de prestigio, pero que no solo apadrinen, sino que compartan y transfieran experiencia y todo lo bueno de ellas al sistema universitario local y a la planta docente e investigadores (know how) . Por ello se recomienda negociar bajo los criterios de igualdad y equidad, aplicando el principio de reciprocidad, para que esas universidades grandes no nos vendan el producto o su prestigio, sino que cumplan su función social también, y que los costos no solo sean asumidos por las universidades salvadoreñas involucradas, o por los estudiantes.

Los sectores productivos, en un contexto de apuesta y riesgo consensuado, deben apoyar a las universidades, no sólo a unas pocas; deben pensar a escala nacional. No es posible mejorar el desarrollo humano, económico y social, si no se le apuesta a la juventud; y las universidades privadas son una vía, que, como ya lo observábamos en las estadísticas, hay un alto porcentaje de la población estudiantil salvadoreña que decide estudiar una carrera profesional. Ya no estamos en guerra, pero muchos son los jóvenes y adultos que aún prefieren las universidades privadas a la nacional. Solo basta darle seguimiento a las estadísticas anuales para verificarlo. Entonces se necesitan respuestas. Hay que buscarlas y darlas. Solamente así la contribución de las universidades privadas será mayor al desarrollo que tanto necesita nuestro país.

\footnotetext{
6 Know-How: Expresión anglosajona utilizada en el comercio internacional. Es una forma de transferencia de tecnología y conocimiento con valor agregado. Habilidades con que cuenta una organización para desarrollar sus funciones productivas o de servicios para denominar los conocimientos preexistentes, no siempre acauémicos, pero que incluyen técnicas e información secreta que propician habilidades y aptitudes particularmente distintivas. Tomado del sitio Web http:/les.wikipedia. org/wiki/
} 